\title{
NOTE ON THE DIAGONALIZATION OF A QUADRATIC LINEAR FORM DEFINED ON THE SET OF SECOND QUANTIZATION FERMION OPERATORS
}

\author{
A. L. KUZEMSKY \\ Laboratory of Theoretical Physics, Joint Institute for Nuclear Research, \\ Head Post Office P.O. Box 79, Moscow, U.S.S.R.
}

A. Pawlikowski

Silesian University, Katowice, Poland

(Received October 19, 1971)

\begin{abstract}
The problem of diagonalization of a quadratic linear form on the set of second quantization fermion operators is discussed and two different cases of diagonalization are indicated. It is shown that the quadratic linear fermion form in the second case may behave as a many-particle operator contrary to the Bose statistics. A particular case of diagonalization of the quadratic linear form on two types of fermion operators is analysed using the Dirac matrix algebra.
\end{abstract}

\section{Introduction}

In paper [10] the diagonalization of the quadratic linear (q.1.) form on the set of second quantization operators $a_{k}$ and $a_{k}^{+}$

$$
L=\sum_{k}\left(F_{k} a_{k}^{+} a_{k}+\varphi_{k} a_{k}+a_{k}^{+} \varphi_{k}^{+}\right), \quad L=L^{+},
$$

was considered. Here $F_{k}, \varphi_{k}, \varphi_{k}^{+}$are certain coefficients, $F_{k}$ being a $c$-number. Attention has been drawn to the fact that when $a_{k}$ and $a_{k}^{+}$satisfy the Bose commutation relations the q.1. form (1.1) is diagonalized simply by the canonical shift transformation. If the operators $a_{k}$ and $a_{k}^{+}$satisfy the Fermi commutation relations, the diagonalizing problem requires special consideration which is just the matter of the present paper.

The expression (1.1) is a Hermitian form and, consequently, it may be diagonalized. However, as will be shown below, since the q.1. form may behave as a many-particle operator, the diagonalization is different for the two following cases.

The first case occurs when the linear fermion sources (i.e. the terms $\varphi_{k} a_{k}+a_{k}^{+} \varphi_{k}^{+}$) are introduced with the aid of anticommutation relations with the initial fields and, simultaneously, by self-anticommuting spinor fields $\varphi_{k}$ and $\varphi_{k}^{+}$. Then the form (1.1) is, in gist, a bilinear fermion form and consequently may be diagonalized by the linear ca- 
nonical transformation [2]. The linear sources in this case may be interpreted as interaction terms between the initial fermion field and the external spinor field. We note that recently a problem similar in some respect was investigated in paper [5]. It consists in diagonalizing the interaction Hamiltonian between a one-mode boson field and one-mode fermion.

The second case occurs when the quantities $\varphi_{k}$ and $\varphi_{k}^{+}$in equation (1.1) are supposed to be $c$-numbers.

In the present note we shall discuss the diagonalization in both cases and investigate the possibility of its practical realization. It should be noted that these questions have not been considered in the monographs available (see e.g. [2]).

\section{Diagonalization in the first case}

Consider the q.1. form (1.1), where $a_{k}$ and $a_{k}^{+}$satisfy the fermion commutation relations and suppose that $\varphi_{k}$ and $\varphi_{k}^{+}$are equal to

$$
\varphi_{k}=\eta_{k} f_{k}, \quad \varphi_{k}^{+}=\eta_{k}^{*} f_{k}^{+},
$$

where $\eta_{k}$ and $\eta_{k}^{*}$ are $c$-numbers and $f_{k}$ and $f_{k}^{+}$are spinors satisfying the following commutation relations:

$$
\left\{f_{k}, f_{l}\right\}=0 ; \quad\left\{f_{k}, f_{k}^{+}\right\}=1, \quad\left\{f_{k}, f_{l}^{+}\right\}=0 \quad \text { for } \quad k \neq l,
$$

Herc $\{\ldots\}$ is an anticommutator. Besides that we need the commutation relations for the fermion fields $a_{k}$ and $f_{k}$. We find them by requiring the terms in the sum (1.1) with distinct indices $k$ to commute:

$$
\left[F_{k} a_{k}^{+} a_{k}+\varphi_{k} a_{k}+a_{k}^{+} \varphi_{k}^{+}, F_{l} a_{l}^{+} a_{l}+\varphi_{l} a_{l}+a_{l}^{+} \varphi_{l}^{+}\right]=0 \text { for } k \neq l,
$$

and hence

$$
\left\{a_{k}, f_{l}^{+}\right\}=0, \quad\left\{a_{k}^{+}, f_{l}^{+}\right\}=0 \quad \text { for } \quad k \neq l .
$$

In addition, we require the fulfilment of the conditions (2.4) for $k=l$. This is a reasonable physical requirement because $a_{k}$ and $f_{k}$ ere related to the distinct fermion fields.

A bilinear hermitian form on the set of second quantization fermion operators can be diagonalized by the linear canonical transformation [2]. We note that the conditions (2.4) permit to consider the operator

$$
\mathrm{Fa}^{+} a+\eta f a+\eta^{*} a^{+} f^{+}
$$

only. In order to diagonalize it we use a linear canonical transformation of the fermion operators $a$ and $f$ to the new operators $b$ and $g$, respectively

$$
\begin{gathered}
a=x_{1} b+x_{2} g^{+}, \quad a^{+}=x_{1}^{*} b^{+}+x_{2}^{*} g, \\
f^{+}=y_{1} b+y_{2} g^{+}, \quad f=y_{1}^{*} b^{+}+y_{2}^{*} g .
\end{gathered}
$$


Here the coefficients $x_{1,2}$ and $y_{1,2}$ have the form

where

$$
x_{1,2}=\frac{\lambda_{1,2}}{\sqrt{|\eta|^{2}+\lambda_{1,2}^{2}}}, \quad y_{1,2}=\frac{\eta}{\sqrt{|\eta|^{2}+\lambda_{1,2}^{2}}},
$$

$$
\lambda_{1,2}=\frac{1}{2}\left(F \pm \sqrt{4|\eta|^{2}+F^{2}}\right)
$$

are the eigenvalues of the matrix which is formed from the coefficients of the equations of motion for the operators $a$ and $f^{+}$. The quantities

$$
\left[\begin{array}{l}
x_{1} \\
y_{1}
\end{array}\right] \text { and }\left[\begin{array}{l}
x_{2} \\
y_{2}
\end{array}\right]
$$

are the eigenvectors of this matrix. If one applies the canonical transformation (2.6) to (2.5), the q.l. form (2.5) reduces to the following expression

$$
\lambda_{1} b^{+} b+\lambda_{2} g g^{+} \text {. }
$$

Thus, the problem of diagonalizing the q.l. form is solved for this case.

\section{Diagonalization in the second case}

This case is more difficult and therefore, to begin with, we assume that the index $k$ in the sum (1.1) takes only one value. The physical meaning of this assumption is that no dispersion occurs. We note that the problem of diagonalizing the q.1. form (1.1) without dispersion has been considered for this case in paper [4] with the aid of both the canonical transformation and the two-time, temperature-dependent Green functions [8], [9].

The operators $a^{+}$and $a$ are defined by the manner in which they act on the vectors $|0\rangle$ and $|1\rangle$ :

$$
\begin{array}{ll}
a|0\rangle=0, & a^{+}|0\rangle=|1\rangle, \\
a|1\rangle=|0\rangle, & a^{+}|1\rangle=0,
\end{array}
$$

and any operator in this space can be expressed as a linear combination of the four linear independent operators $a, a^{+}, a^{+} a, 1$. Let us introduce a unitary operator $U$ which diagonalizes the q.l. form and such that $U a U^{+}=b, U a^{+} U^{+}=b^{+}$. Then we have

$$
\begin{aligned}
b & =\tau_{1} a^{+} a+\tau_{2} a+\tau_{3} a^{+}, \\
b^{+} & =\tau_{1} a^{+} a+\tau_{3}^{*} a+\tau_{2}^{*} a^{+}, \\
b^{+} b & =\left(\tau_{1}^{2}+\left|\tau_{2}\right|^{2}+\left|\tau_{3}\right|^{2}\right) a^{+} a+\tau_{1} \tau_{3}^{*} a+\tau_{1} \tau_{3} a^{+},
\end{aligned}
$$

where $\tau_{1}, \tau_{2}, \tau_{3}$ are certain coefficients and $\tau_{1}^{*}=\tau_{1}$. The resolution of the system (3.2) with respect to $a^{+}$and $a$ enables to express $\tau_{1}, \tau_{2}, \tau_{3}$ in terms of the parameters $F$, $\varphi$ and $\varphi^{*}$ of the problem. The characteristic equation in this case is

$$
\lambda^{2}-|f|^{2}=0, \quad \lambda= \pm|f| \text {. }
$$


If the characteristic roots are known, one can evaluate the eigenvectors of the matrix (1.1) in the basis (3.1) and, consequently, find the diagonalizing matrix [1]. We note that the fermion operators without index are, in principle, the Pauli operators [6].

One can consider the situation in the same way when the index $k$ in equation (1.1) is equal to $k=1$ and $k=2$.

In the basis

$$
L=F_{1} a_{1}^{+} a_{1}+\varphi_{1} a_{1}+\varphi_{1}^{*} a_{1}^{+}+F_{2} a_{2}^{+} a_{2}+\varphi_{2} a_{2}+\varphi_{2}^{*} a_{2}^{+} .
$$

$$
|00\rangle=|0\rangle|0\rangle, \quad|10\rangle=|1\rangle|0\rangle, \quad|01\rangle=|0\rangle|1\rangle, \quad|11\rangle=|1\rangle|1\rangle
$$

any operator can be expressed as a linear combination of the 16 operators

$$
\begin{aligned}
b=U a U^{+}= & c_{1} a_{1}^{+} a_{1}+c_{2} a_{1}+c_{3} a_{1}^{+}+c_{4} a_{2}^{+} a_{2}+c_{5} a_{2}+c_{6} a_{2}^{+}+ \\
& +c_{7} a_{1} a_{2}+c_{8} a_{2}^{+} a_{1}+c_{9} a_{1}^{+} a_{2}+c_{10} a_{2}^{+} a_{1}+c_{11} a_{1} a_{2}^{+} a_{2}+ \\
& +c_{12} a_{1}^{+} a_{2}^{+} a_{2}+c_{13} a_{2}^{+} a_{1}^{+} a_{1}+c_{14} a_{2} a_{1}^{+} a_{1}+c_{15} a_{1}^{+} a_{1} a_{2}^{+} a_{2}+c_{16} .
\end{aligned}
$$

The matrix $\bar{L}$ in the basis (3.5) has the form

$$
\bar{L}=\left[\begin{array}{cccc}
0 & f_{1} & f_{2} & 0 \\
f_{1}^{*} & F_{1} & 0 & -f_{2} \\
f_{2}^{*} & 0 & F_{2} & f_{1} \\
0 & -f_{2}^{*} & f_{1}^{*} & F_{1}+F_{2}
\end{array}\right] .
$$

and then the characteristic equation is

$$
\begin{aligned}
& \lambda^{4}-2\left(F_{1}+F_{2}\right) \lambda^{3}+\left\{\left(F_{1}+F_{2}\right)^{2}+F_{1} F_{2}-2\left(\left|\varphi_{1}\right|^{2}+\left|\varphi_{2}\right|^{2}\right)\right\} \lambda^{2}+ \\
&+\left(F_{1}+F_{2}\right)\left(-F_{1} F_{2}+2\left(\left|\varphi_{1}\right|^{2}\right.\right.\left.\left.+\left|\varphi_{2}\right|^{2} \lambda+\left|\varphi_{1}\right|^{2}+\left|\varphi_{2}\right|^{2}\right)\right)- \\
&-\left(F_{1}+F_{2}\right)\left(\left|\varphi_{1}\right|^{2} F_{2}+\left|\varphi_{2}\right| F_{1}\right)=0 .
\end{aligned}
$$

In the general case, one can see that for $k>1$ the q.1. form defined on the set of fermion operators may not be a one-particle operator, i.e. may not have the form $L^{\prime}=\sum_{\alpha} \varepsilon_{\alpha} b_{\alpha}^{+} b_{\alpha}$.

In certain cases the terms $a_{1}^{+} a_{1} a_{2}^{+} a_{2}$ may be excluded. The criterion for this will be given in the next section. A more general situation, when $k>2$, may be considered in a similar way [7].

\section{The use of the Dirac matrix algebra}

The diagonalization of the q.1. forms (2.5) and (3.4) defined on two types of fermion operators can be simplified if one uses the Clifford algebra [3] of the Dirac matrices $\Gamma_{\alpha \beta}$ which are expressed through the Kronecker product of the Pauli matrices $\sigma_{\alpha}$ [1]

$$
\Gamma_{\alpha \beta}=\sigma_{\alpha} \cdot \sigma_{\beta}, \quad \alpha, \beta=0,1,2,3, \quad \sigma_{0}=\left[\begin{array}{ll}
1 & 0 \\
0 & 1
\end{array}\right] .
$$


Any four-dimensional matrix $\bar{C}$ can be written as

$$
\bar{C}=\sum_{\alpha, \beta} g_{\alpha \beta} \Gamma_{\alpha \beta}, \quad \text { where } \quad g_{\alpha \beta}=\frac{1}{4} \operatorname{Sp}\left\{\bar{C} \cdot \Gamma_{\alpha \beta}\right\}
$$

Let us introduce the vacuum state and the basis in the following way

$$
\left.\begin{array}{cl}
a_{1}|00\rangle=0, \quad a_{2}|00\rangle=0, \quad\langle 00 \mid 00\rangle=1, \\
|00\rangle=|0\rangle, \quad, \quad|01\rangle=a_{2}^{+}|00\rangle=|2\rangle, \\
|10\rangle=a_{1}^{+}|00\rangle=|1\rangle, \quad|11\rangle=a_{1}^{+} a_{2}^{+}|00\rangle=|3\rangle .
\end{array}\right\}
$$

In this basis we have

$$
a_{1}=\left[\begin{array}{rrrr}
0 & 1 & 0 & 0 \\
0 & 0 & 0 & 0 \\
0 & 0 & 0 & 1 \\
0 & 0 & 0 & 0
\end{array}\right], \quad a_{1}^{+}=\left(a_{1}\right)^{+}, \quad a_{2}=\left[\begin{array}{rrrr}
0 & 0 & 1 & 0 \\
0 & 0 & 0 & -1 \\
0 & 0 & 0 & 0 \\
0 & 0 & 0 & 0
\end{array}\right], \quad a_{2}^{+}=\left(a_{2}\right)^{2}
$$

From (4.5) it follows that

$$
\begin{array}{ll}
\Gamma_{01}=a_{1}+a_{1}^{+}, & \Gamma_{10}=\left(1-2 a_{1}^{+} a_{1}\right)\left(a_{2}^{+}+a_{2}\right), \\
\Gamma_{02}=i\left(a_{1}^{+}-a_{1}\right), & \Gamma_{20}=i\left(1-2 a_{1}^{+} a_{1}\right)\left(a_{2}^{+}-a_{2}\right), \\
\Gamma_{03}=\left(1-2 a_{1}^{+} a_{1}\right), & \Gamma_{30}=1-2 a_{2}^{+} a_{2}, \\
\Gamma_{13}=a_{2}+a_{2}^{+}, & \Gamma_{31}=\left(a_{1}^{+}+a_{1}\right)\left(1-2 a_{2}^{+} a_{2}\right), \\
\Gamma_{23}=i\left(a_{2}^{+}-a_{2}\right), & \Gamma_{32}=i\left(a_{1}^{+}-a_{1}\right)\left(1-2 a_{2}^{+} a_{2}\right), \\
\Gamma_{12}=i\left(a_{1}^{+}+a_{1}\right)\left(a_{2}^{+}+a_{2}\right), & \Gamma_{11}=\left(a_{1}^{+}-a_{1}\right)\left(a_{2}^{+}+a_{2}\right), \\
\Gamma_{22}=-\left(a_{1}^{+}+a_{1}\right)\left(a_{2}^{+}-a_{2}\right), & \Gamma_{21}=i\left(a_{1}^{+}-a_{1}\right)\left(a_{2}^{+}-a_{2}\right), \\
& \Gamma_{33}=\left(1-2 a_{1}^{+} a_{1}\right)\left(1-2 a_{2}^{+} a_{2}\right) .
\end{array}
$$

In the basis (4.4) the q.1. form (3.4) is represented by the matrix

$$
\bar{L}=\left[\begin{array}{ccc}
L_{00} & \ldots & L_{03} \\
\ldots & \ldots & \ldots \\
L_{30} & \ldots & L_{33}
\end{array}\right]
$$

which is diagonalized by the unitary matrix $U$

$$
\bar{L}^{\prime}=U^{-1} \bar{L} U=\left[\begin{array}{cccc}
E_{0} & & 0 \\
& E_{1} & & \\
& & E_{2} & \\
0 & & E_{3}
\end{array}\right]
$$


The transformed operator is

$$
b_{j}=U^{+} a_{j} U, \quad b_{j}^{+}=U^{+} a_{j}^{+} U, \quad j=1,2 .
$$

Now, expressing the matrices of the operators $U, L^{\prime}, b, b^{+}$in terms of $\Gamma_{\alpha \beta}$ one can find how these operators are represented through the initial operators. After diagonalization, the q.1. form (3.4) is written as a linear combination of the values $\Gamma_{00}, \Gamma_{03}, \Gamma_{30}$, $\Gamma_{33}$. One can point out a simple rule indicating when terms of the type $a_{1}^{+} a_{1} a_{2}^{+} a_{2}$ are excluded, by putting the coefficients for $\Gamma_{33}$ equal to zero

$$
C_{33}=\frac{1}{4} \operatorname{Sp}\left(\Gamma_{33} L^{\prime}\right)=0 \text {. }
$$

If the ground state is chosen properly, the equality $E_{0}+E_{3}=E_{1}+E_{2}$ holds.

\section{Concluding remarks}

The diagonalization of the q.1. form defined on the second quantization fermion operators is thus more complicated compared with the Bose statistics. In practice, the diagonalization differs largely in the two cases mentioned.

In the first case the linear terms may be interpreted as an interaction of the initial fermion field with an external spinor field and the q.l. form is easily diagonalized by a linear canonical transformation.

In the second case the diagonalization is more complicated since the fermion operators with different indices anticommute and, consequently, the whole sum must be diagonalized simultaneously. This fact can be interpreted as the presence of a certain interaction in the system.

Such a distinction between the Bose and Fermi systems is associated with the symmetry properties of their wave functions. For a Bose system the addition of a particle leads to the displacement of the one-particle energies only, while in the case of Fermi statistics the whole system must be reconstructed. Moreover, the one-particle energies may be nonadditive. Thus, one can say that the q.l. fermion form may be a many-particle operator unlike it is in the Bose statistics. It is also a lead in distinguishing between various types of diagonalizations. This results in different diagonalization procedures.

We would like to thank Professor D. N. Zubarev for valuable discussions and Professor E. Czerwonko, Dr. Yu. A. Tserkovnikov and Dr. M. Yu. Novikov for useful remarks.

\section{REFERENCES}

[1] Bellman, R., Introduction to matrix analysis, McGraw-Hill Book Comp., New York, Toronto, London, 1960.

[2] Berezin, F. A., Methods of second quantization, Nauka, Moscow, 1965.

[3] Corson, E. M., Introduction to tensors, spinors, and relativistic wave-equation, Blackie and Son Ltd., London, 1953. 
[4] ter Haar, D., W. E. Parry, Phys. Lett. 1 (1962), 145.

[5] Trubatch, S. L., Amer. Journ. Phys. 38 (1970), 331.

[6] Tyablikov, S. V., Methods for quantum theory of magnetism, Plenum Press, New York, 1967.

[7] Wilkinson, J. H., The algebraic eigenvalue problem, Clarendon Press, Oxford, 1965.

[8] Zubarev, D. N., Usp. Fiz. Nauk 71 (1960), 71.

[9] -, Non-equilibrium statistical thermodynamics, Nauka, Moscow, 1971.

[10] Zubarev, D. N., A. L. Kuzemsky, K. Walasek, Theor. Math. Phys. 6 (1970), 280. 